\title{
SOLUTION OF THE LARGE MINIMUM COMPLIANCE PROBLEM USING BILEVEL OPTIMIZATION
}

\author{
Abdelilah Makrizi ${ }^{1}$ and Bouchaïb Radi ${ }^{2}$ \\ ${ }^{1}$ Department of Mathematics, CRMEF Beni Mellal-Khenifra, Khouribga, Morocco \\ a makrizi@yahoo.fr \\ ${ }^{2}$ Faculty of sciences and technics, Hassan Premier University, Settat, Morocco \\ bouchaib.radi@yahoo.fr
}

\section{ABSTRACT}

The topology optimization problem have a great industrial interest. Using the subdomains method, we have formulated the decomposed topology optimization problem as a bilevel one. In this paper, we reformulate our bilevel problem as a single level optimization problem by replacing the lower level optimization problem with its KKT optimality conditions, we give also a new algorithm and numerical results.

\section{Indexing terms/Keywords}

Topology optimization; subdomains method parallel computing; bilevel optimization; Karush-Kuhn-Tucker conditions.

\section{Academic Discipline And Sub-Disciplines}

Optimization, structural optimizatuiion.

\section{MATHEMATICS SUBJECT CLASSIFICATION (2010)}

74P05, 65M55, 65Y05, 91A65, 90C46.

\section{TYPE (METHOD/APPROACH)}

A new approach for solving minimum compliance problem based bilevel analysis for the decomposed topology optimization problem.

\section{INTRODUCTION}

The problem of topology optimization is typically regarded as material distribution problem for the optimal shape design [8]. The distribution of the material is limited to the design domain, which forms part of a larger domain which can include areas prescribed to be solid or void. The problem considered in this paper is the minimal compliance problem. Minimizing compliance turned out to be a standard problem in topology optimization [3]. This problem aims at the design of the stiffest (or least compliant) structure under a given fixed load, possible support conditions and restriction on the volume of the used material.

We specify the problem mathematically in the SIMP approach as follows [8]:

$$
\left\{\begin{array}{c}
\min _{\rho} l(u) \\
a_{\rho}(u, v)=l(v) \quad \forall v \in U \subseteq H_{0}^{1}(\Omega)^{d} \\
E_{i j k l}(x)=\rho^{p}(x) E_{i j k l}^{0}
\end{array}\right.
$$

with the following constraints on $\rho$ :

$\int_{\Omega} \rho(x) d \Omega \leq V, 0<\rho_{\min }<\rho(x) \leq 1, \forall x \in \Omega$

$U$ is the set of admissible displacements, $V$ is a limit on the amount of material at our disposal, ${ }^{E_{i j k l}^{0}}$ represents the material properties of a given isotropic material, $\rho$ which is interpreted as a density of material is the design variable and $p$ is the penalty factor which penalizes intermediate densities in order to end up with (nearly) 'solid and void' distributions, where:

$a_{\rho}(u, v)=\int_{\Omega} \rho^{p}(x) E_{i j k l}^{0} \varepsilon_{i j}(u) \varepsilon_{k l}(v) d \Omega$.

and

$l(v)=\int_{\Omega} f v d \Omega$ 
$f$ is the vector of volume forces acting on the body, Topology optimization has for a considerable time been applied successfully in the automotive industry, but still has not become a mainstream technology for the design of large structures and complex materials like aircraft components. The explanation for this is partly to be sought in the larger problem sizes.

The Domain Decomposition Method (DDM) is one of the most effective parallel methods for large scale problems due to the fact that it provides a high level of concurrency and is simple to implement on most modern parallel computers. The principle of the method is to split the original domain of computation in smaller simpler subdomains, which may or may not overlap. Next, the original problem can be reformulated upon each subdomain, yielding a family of subproblems of reduced size that are coupled one to another through the values of the unknown solution at subdomain interfaces.

\section{PROBLEM STATEMENT}

Suppose that $\Omega$ is partitioned into two nonoverlapping subdomains $\Omega_{1}$ and $\Omega_{2}$ with interface $\Gamma_{0}$ i.e. $\Gamma_{0}=\overline{\Omega_{1}} \cap \overline{\Omega_{2}}$. Let $\Gamma_{i}=\overline{\Omega_{i}} \cap \Gamma$ and $u_{i}=u / \Omega_{i} i=1,2$. Applying domain decomposition method in minimum compliance problem (1), we have obtained the following optimization problem: [1]

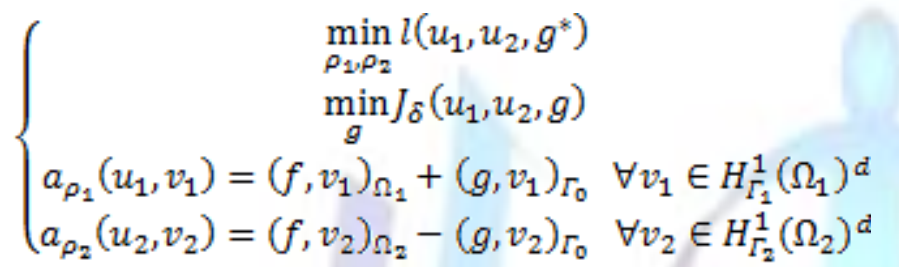

with the constraints on $\rho_{1}, \rho_{2}$ :

$\sum_{i=1}^{2} \int_{\Omega_{i}} \rho_{i}(x) d \Omega_{i} \leq V, \quad 0<\rho_{\min } \leq \rho_{i}(x) \leq 1 \quad \forall x \in \Omega_{i}, i=1,2$.

where

$I_{\delta}\left(u_{1}, u_{2}, g\right)=\frac{1}{2} \int_{\Gamma_{0}}\left(u_{1}-u_{2)}{ }^{2} d \Gamma_{0}+\frac{\delta}{2} \int_{\Gamma_{0}} g^{2} d \Gamma_{0}\right.$

and " $\mathrm{g}$ " $=\mathrm{g}$ opt" is the unique choice of $g$ for which $\left(g, u_{1}\right)_{\Gamma_{0}}=\left(g, u_{2}\right)_{\Gamma_{0}}$ i.e.

$\int_{\Gamma_{0}} g u_{1} d \Gamma_{0}=\int_{\Gamma_{0}} g u_{2} d \Gamma_{0}$

(see [1] Remark 3.7)

In [2] we have proved that our problem (2), which involve an hierarchical making process, is a bilevel programming problem. In fact, on one hand, the functions $I\left(u_{1} ; u_{2} ; g\right)$ and $I_{\delta}\left(u_{1}, u_{2}, g\right)$ can't be minimized simultaneously, so, bicriteria optimization is no more suitable, and the hierarchical nature of the two levels is a natural justification for this choice.

On the other hand, structural optimization problems have an inherent bilevel form. The upper level objective function measures some performance of the structure, such as the stiffness in our example. The lower level problem describes the behavior of the structure given the choices of the design variables and the external forces acting on it.

The theory of bilevel programming focuses on forms of optimality conditions and complexity results, the complexity of the problem has been addressed by a number of authors, it has been proved that even the linear programming problem, where all the involved functions are affine, is a strongly NP-hard problem, in addition, due to the hierarchical structure the BLPP is not convex and not differentiable.

The formulation of optimality conditions for bilevel programming problems usually starts with a suitable reformulation of the problem as a one-level one by replacing the lower level optimization problem with its KKT optimality conditions, which are necessary and sufficient for defining the optimum of the inner level problem only under convexity condition and a first order constraint qualification. When the inner problem constraints are non convex, the KKT conditions are only necessary. A further difficulty arises in locating the global optimum of the resulting single level problem after the KKT transformation, the bilinear nature of complementarity condition introduce nonconvexities even if the original problem is linear.

A topology optimization problem is an optimization problem where a generalized equation(modeling an equilibrium) arises among the constraints. This new class of optimization problems which play an important role in mechanics, is known as 
generalized bilevel programming problems, or mathematical programs with equilibrium constraints or (MPEC) [5] [6], and largely unknown to the structural optimization community.

The MPEC problems coming from the topology optimization of structures, solids and fluids violates standard non linear programming constraint qualifications, and even the novel qualification, such as the strict complementarity conditions, or strong regularity assumptions. Therefore, Evgrafov [9] proposed approximation techniques for the numerical solution of such problems. As mentioned above, one tool often used to reformulate the bilevel programming problem as an one level problem are the Karush-Kuhn-Tucker conditions. If a regularity condition is satisfied for the lower level problem, then the KKT conditions are necessary optimality conditions. They are also sufficient in the case when the lower level problem is convex, this is the approach used in this paper.

The paper is organized in the following way: in section 3 the mathematical analysis of the bilevel formulation is given, and a new single level optimization problem is deduced, and an algorithm is proposed for numerical results in section 4.

\section{REFORMULATION OF THE DECOMPOSED TOPOLOGY OPTIMIZATION PROBLEM USING THE KUHN-TUCKER APPROACH}

Consider the lower level problem:

$$
\left\{\begin{array}{c}
\min _{g} J_{\delta}\left(u_{1}, u_{2}, g\right) \\
a_{\rho_{1}}\left(u_{1}, v_{1}\right)=\left(f, v_{1}\right)_{\Omega_{1}}+\left(g, v_{1}\right)_{\Gamma_{0}} \\
a_{\rho_{2}}\left(u_{2}, v_{2}\right)=\left(f, v_{2}\right)_{\Omega_{2}} \in\left(g, v_{2}\right)_{\Gamma_{0}} \quad \forall v_{2} \in H_{\Gamma_{2}}^{1}\left(\Omega_{1}\right)^{d}
\end{array}\right.
$$

The conventional solution approach to the BLPP is, as mentioned earlier, to transform the original two level problem into a single level one by replacing the lower optimization problem with the set of equations that define its KKT optimality conditions.

Noting that, the KKT optimality conditions are necessary and sufficient for definig the optimum of the inner level problem only under convexity condition ans a first order constraint qualifications. When the inner problem constraints are nonconvex, the KKT conditions are only necessary. Without inequality constraints in the lower level problem, the resulting single level problem after the KKT transformation lacks complementarity conditions which introduce nonconvexities even if the original problem is linear.

In our case, the convex nature of the inner problem (3) enable us to apply the Kuhn-Tucker approach to transform the bilevel problem (2) into a single one and derive the KKT optimality conditions.

For the lower level problem (3) define the Lagrangian:

$L_{\text {low }}\left(u_{1}, u_{2}, g, \lambda_{1}, \lambda_{2}\right)=J_{\delta}\left(u_{1}, u_{2}, g\right)-a_{\rho_{1}}\left(u_{1}, \lambda_{1}\right)+\left(f, \lambda_{1}\right)_{\Omega_{1}}+\left(g, \lambda_{1}\right)_{\Gamma_{0}}$

$a_{\rho_{2}}\left(u_{2}, \lambda_{2}\right)+\left(f, \lambda_{2}\right)_{\Omega_{2}}-\left(g, \lambda_{2}\right)_{\Gamma_{0}}$

where

$\left(u_{1}, u_{2}, g_{1}, \lambda_{1}, \lambda_{2}\right) \in H_{\Gamma_{1}}^{1}\left(\Omega_{1}\right)^{d} \times H_{\Gamma_{2}}^{1}\left(\Omega_{2}\right)^{d} \times L^{2}\left(\Gamma_{0}\right)^{d} \times H_{\Gamma_{1}}^{1}\left(\Omega_{1}\right)^{d} \times H_{\Gamma_{2}}^{1}\left(\Omega_{2}\right)^{d}$

Then, a necessary and sufficient condition for $g_{*}$ to be an optimal solution to the inner level problem is that:

$\frac{\partial L_{\text {lox }}\left(u_{1}^{*}, u_{2}^{*} g^{*} \lambda_{1}^{*} \lambda_{2}^{*}\right)}{\partial g}=0$

gives the optimality condition

For $\mathrm{i}=1,2$

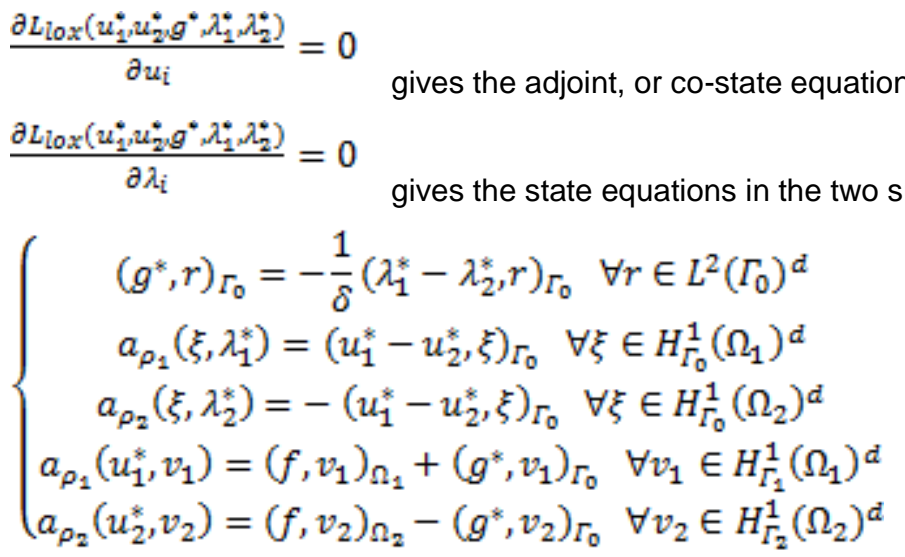


Journal of Advances in Mathematics

The equations (4) are the Kuhn-Tucker necessary optimality conditions for the problem (3), and under the convexity assumptions of the Lagrangian, they are sufficient conditions for $\left(u_{1}^{*}, u_{2}^{*}, g^{*}\right)$ to be an optimal solution of the problem (3). Given that $f \circ g$ is convex when $f$ and $g$ are convex and $f$ not decreasing, then the function $x \rightarrow\|x\|^{2}$ is convex because $x \rightarrow\|x\|$ and $x \rightarrow x^{2}$ are convex and $x \rightarrow x^{2}$ increases for $x \geq 0$, therefore the objective function ${ }^{I \delta}$ is convex in $\mathrm{u}_{1}$ $\mathrm{u}_{2}$ and $\mathrm{g}$.

The convexity of the Lagrangian ${ }^{L_{\text {low }}}$ in $\mathrm{u}_{1}, \mathrm{u}_{2}$ and $\mathrm{g}$ then follows from the fact that ${ }^{I \delta}$ is convex and the state equations are linear.

It follows that a necessary condition for $\left(\overline{\rho_{1}}, \overline{\rho_{2}}, \overline{u_{1}}, \overline{u_{2}}, \overline{\lambda_{1}}, \overline{\lambda_{2}}, g\right)$ to be an optimal solution of the bilevel problem (2), $\left(\overline{u_{1}}, \overline{u_{2}}, \overline{\lambda_{1}}, \overline{\lambda_{2}}, g\right)$ must satisfy the above conditions at fixed $\left(\rho_{1}, \rho_{2}\right)=\left(\overline{\rho_{1}}, \overline{\rho_{2}}\right)$, thus the bilevel programming problem (2) is transformed into a single level problem of the form:

$$
\left\{\begin{array}{c}
\min _{\rho_{1}, \rho_{2}} l\left(u_{1}, u_{2}, g\right) \\
a_{\rho_{1}}\left(u_{1}, v_{1}\right)=\left(f, v_{1}\right)_{\Omega_{1}}+\left(g, v_{1}\right)_{\Gamma_{0}} \quad \forall v_{1} \in H_{\Gamma_{1}}^{1}\left(\Omega_{1}\right)^{d} \\
a_{\rho_{2}}\left(u_{2}, v_{2}\right)=\left(f, v_{2}\right)_{\Omega_{2}}-\left(g, v_{2}\right)_{\Gamma_{0}} \forall v_{2} \in H_{\Gamma_{2}}^{1}\left(\Omega_{2}\right)^{d} \\
a_{\rho_{1}}\left(\xi, \lambda_{1}\right)=\left(u_{1}-u_{2}, \xi\right)_{\Gamma_{0}} \forall \xi \in H_{\Gamma_{0}}^{1}\left(\Omega_{1}\right)^{d} \\
a_{\rho_{2}}\left(\xi, \lambda_{2}\right)=-\left(u_{1}-u_{2}, \xi\right)_{\Gamma_{0}} \forall \xi \in H_{\Gamma_{0}}^{1}\left(\Omega_{2}\right)^{d} \\
(g, r)_{\Gamma_{0}}=-\frac{1}{\delta}\left(\lambda_{1}-\lambda_{2}, r\right)_{\Gamma_{0}} \quad \forall r \in L^{2}\left(\Gamma_{0}\right)^{d}
\end{array}\right.
$$

with the constraints on $\rho_{1}, \rho_{2}$ :

$\sum_{i=1}^{2} \int_{\Omega_{i}} \rho_{i}(x) d \Omega_{i} \leq V, \quad 0<\rho_{\text {min }} \leq \rho_{i}(x) \leq 1 \quad \forall x \in \Omega_{i}, i=1,2$.

\section{PROPOSED ALGORITHM FOR NUMERICAL RESULTS}

\subsection{The optimality system}

Define the Lagrange function for the single level problem (5) as follows:

$\mathrm{I}=1,2$

$$
\begin{aligned}
& L\left(u_{v} \rho_{u}, g, \bar{u}_{v} \lambda_{u}, \tilde{\lambda}_{u}, \tilde{r}_{s} \Lambda, \lambda_{u}^{+}(x), \lambda_{L}^{-}(x)\right) \\
& =l\left(u_{1}, u_{2}, g\right)-\left(a_{\rho_{1}}\left(u_{1}, \overline{u_{1}}\right)-\left(f, \overline{u_{1}}\right)_{\Omega_{1}}-\left(g, \overline{u_{1}}\right)_{\Gamma_{0}}\right) \\
& -\left(a_{\rho_{2}}\left(u_{2}, \overline{u_{2}}\right)-\left(f, \overline{u_{2}}\right)_{\Omega_{2}}+\left(g, \overline{u_{2}}\right)_{\Gamma_{0}}\right)-\left(a_{\rho_{1}}\left(\lambda_{1}, \widetilde{\lambda_{1}}\right)-\left(u_{1}-u_{2}, \widetilde{\lambda_{1}}\right)_{\Gamma_{0}}\right) \\
& -\left(a_{\rho_{2}}\left(\lambda_{2}, \widetilde{\lambda_{2}}\right)+\left(u_{1}-u_{2}, \widetilde{\lambda_{2}}\right)_{\Gamma_{0}}\right)-\left((g, \tilde{r})_{\Gamma_{0}}+\frac{1}{\delta}\left(\lambda_{1}-\lambda_{2}, \tilde{r}\right)_{\Gamma_{0}}\right) \\
& +\Lambda\left(\sum_{i=1}^{2} \int_{\Omega_{l}} \rho_{i}(x) d \Omega_{l}-V\right)+\sum_{i=1}^{2} \int_{\Omega_{l}} \lambda_{l}^{+}(x)\left(\rho_{i}(x)-\Lambda\right) d \Omega_{l} \\
& +\sum_{i=1}^{2} \int_{\Omega_{l}} \lambda_{l}^{-}(x)\left(\rho_{\min }-\rho_{i}(x)\right) d \Omega_{l}
\end{aligned}
$$

where, ${ }^{\overline{u_{l}}}$ are the Lagrange multipliers for the equilibrium constraints, ${ }^{\tilde{\lambda}_{l}}$ and $\tilde{\tilde{r}}$ are also Lagrange multipliers corresponding to the co-state equations and optimality condition for the lower level problem, the Lagrange multipliers $\Lambda, \lambda_{i}^{+}(x), \lambda_{i}^{-}(x)$ are related to the constraints in $\rho_{i}(l=1,2)$

The KKT optimality condition for the problem(5) can be formally interpreted as stationary points of $L(x, y, y x, y x, y x, x, y)$. 
$\frac{\partial L}{\partial u_{i}}=0 \Rightarrow u_{i}=\bar{u}_{i}$

$\frac{\partial L}{\partial \lambda_{L}}=0 \Rightarrow a_{\rho_{L}}\left(\widetilde{\lambda_{L}}, \widetilde{\lambda}_{\mathrm{L}}\right)=(-1)^{\mathrm{L}} \frac{1}{\delta}\left(\tilde{r}, \widetilde{\lambda}_{\mathrm{L}}\right)_{\Gamma_{0}} \forall \widetilde{\lambda}_{\mathrm{L}} \in H_{\Gamma_{\mathrm{i}}}^{1}\left(\Omega_{\mathrm{L}}\right)^{d}$

$\frac{\partial L}{\partial g}=0 \Rightarrow\left(2 g_{0}, u_{1}-u_{2}\right)_{\Gamma_{0}}=\left(g_{0}, \tilde{r}\right)_{\Gamma_{0}} \quad \forall g_{0} \in L^{2}\left(\Gamma_{0}\right)^{d}$

The two last equations yields

$\widetilde{\lambda_{i}}=\lambda_{i}$

then combining (6) and (9), we have the following optimality condition for $\rho_{t}$ :

$\frac{\partial L}{\partial \rho_{i}}=0 \Rightarrow p \rho_{i}(x)^{p-1} E_{i j k l}^{0}\left(\varepsilon_{i j}\left(u_{i}\right) \varepsilon_{k l}\left(u_{i}\right)+\varepsilon_{i j}\left(\lambda_{i}\right) \varepsilon_{k l}\left(\lambda_{i}\right)\right)=\Lambda+\lambda_{i}^{+}-\lambda_{i}^{-}$

the remainder of this optimality system is as follows:

$\frac{\partial L}{\partial \bar{u}_{i}}=0 \Rightarrow a_{\rho_{i}}\left(u_{i}, v_{\mathrm{L}}\right)=\left(f, v_{\mathrm{L}}\right)_{\Omega_{\mathrm{l}}}+(-1)^{\mathrm{a}+1}\left(g, v_{\mathrm{L}}\right)_{\Gamma_{0}} \quad \forall v_{\mathrm{L}} \in H_{\Gamma_{\mathrm{L}}}^{1}\left(\Omega_{\mathrm{l}}\right)^{d}$

$\frac{\partial L}{\partial \widetilde{\lambda}_{L}}=0 \Rightarrow a_{\rho_{L}}\left(\xi, \lambda_{L}\right)=(-1)^{i+1}\left(u_{1}-u_{2}, \xi\right)_{\Gamma_{0}} \quad \forall \xi \in H_{\Gamma_{0}}^{1}\left(\Omega_{L}\right)^{d}$

$\frac{\partial L}{\partial \tilde{r}}=0 \Rightarrow(g, r)_{\Gamma_{0}}=-\frac{1}{\delta}\left(\lambda_{1}-\lambda_{2}, r\right)_{\Gamma_{0}} \forall r \in L^{2}\left(\Gamma_{0}\right)^{d}$

and the conditions related to the bound's constraints

$\Lambda\left(\sum_{i=1}^{2} \int_{\Omega_{l}} \rho_{i}-V\right)=0$

$\lambda_{i}^{-}\left(\rho_{\min }-\rho_{i}\right)=0$

$\lambda_{i}^{+}\left(\rho_{i}-1\right)=0$

with the nonnegativity of the Lagrange multipliers:

$\Lambda \geq 0, \quad \lambda_{\mathrm{L}}^{-} \geq 0, \quad \lambda_{\mathrm{i}}^{+} \geq 0$

For intermediate densities, namely, bound constraints are not active $\left(\rho_{\min }<\rho_{i}<1\right)$, the corresponding multipliers $\left(\lambda_{L}^{-}, \lambda_{L}^{+}\right)$are equal to zero and the optimality condition (10) simplifies to:

$p \rho_{i}(x)^{p-1} E_{i j k l}^{0}\left(\varepsilon_{i j}\left(u_{i}\right) \varepsilon_{k l}\left(u_{i}\right)+\varepsilon_{i j}\left(\lambda_{i}\right) \varepsilon_{k l}\left(\lambda_{i}\right)\right)=\Lambda$

then we have the following update scheme for the density $\rho_{i}[8, p .10]$ :

$\rho_{l, k+1}=\left\{\begin{array}{c}\max \left\{(1-\zeta)_{\rho_{l, k},} \rho_{\min }\right\} \text { if } \rho_{h, k} B_{k}^{\eta} \leq \max \left\{(1-\zeta)_{\left.\rho_{l, k}, \rho_{\min }\right\},},\right. \\ \min \left\{(1+\zeta)_{\left.\rho_{l, k}, 1\right\},} \text { if } \min \left\{(1+\zeta)_{\left.\rho_{l, k}, 1\right\}} \leq \rho_{l, k} B_{k}^{\eta},\right.\right. \\ \rho_{l, k} B_{k}^{\eta} \text { otherwise. }\end{array}\right.$

$\rho_{\iota_{k} k}$ denotes the value of the density variable in the subdomain ${ }^{\Omega_{k}}$ at iteration step $k$, and ${ }^{B} k$ is given by the expression

$B_{k}=\Lambda_{k}^{-1} p \rho_{i}(x)^{p-1} E_{i j k l}^{0}\left(\varepsilon_{i j}\left(u_{l_{z} k}\right) \varepsilon_{k l}\left(u_{i_{l} k}\right)+\varepsilon_{i j}\left(\lambda_{i_{k} k}\right) \varepsilon_{k l}\left(\lambda_{l_{l} k}\right)\right)$ 
where ${ }^{u_{L_{z} k}}$ and ${ }^{\lambda_{L_{2} k}}$ are determined from the equations (11) and (12) respectively, ${ }^{\eta}$ is a tuning parameter and ${ }^{\zeta}$ a move limit, their values are chosen by experiment. A typical useful value of ${ }^{\eta}$ and ${ }^{\bar{\zeta}}$ is 0.5 and 0.2 respectively. The Lagrange multiplier for the volume constraint, ${ }^{{ }_{k}}$ is determined at each iteration using a bisectioning algorithm.

\subsection{The proposed algorithm}

The algorithm described in [8, p.14] to solve the compliance topology design problem consists of the following modules:

1. Finite element analysis

2. Sensitivity analysis

3. Mesh independency filter

4. Update design variables

In our algorithm, only FEA and sensitivity analysis are parallelized, because on one hand, the main part of the computational time is devoted to the solution of the equilibrium equations. However, the effort required to update design variables at each optimization iteration is small compared to the corresponding finite element solve, hence, the FEA is the most important part of the entire solution algorithm to parallelize.

On the other hand, mesh independency is not parallelized, since filtering is not local by nature and requires the average of the sensitivities over several elements. Also, calculations related to sensitivity filtering are negligible compared to those required for the solution of the equilibrium equation and calculation of the element sensitivities.

Because the Lagrange multiplier ${ }^{\Lambda_{k}}$ used to calculate ${ }^{B_{k}}$ in (16) is a global parameter, and when filtering (which is not parallelized) is applied, the optimization update (15) won't be parallelized. The equations (11) (12) (13) are coupled, that's why a gradient method is used to uncouple the search of local displacements $u_{i}(i=1,2)$, and a loop on the control variable $^{g}$ satisfies the continuity of the displacement on the interface $u_{1}=u_{2}$ on $\Gamma_{0}$.

To update $g$ let:

$M_{\delta}(g)=J_{\delta}\left(u_{1}(g),{ }^{u_{2}}(g), g\right)$

where, for given $\mathrm{g}$,

$u_{i}(g): g \in L^{2}\left(\Gamma_{0}\right)^{d} \rightarrow H_{\Gamma_{i}}^{1}\left(\Omega_{i}\right)^{d} i=1,2$

are defined as the solutions of (11).

Compute the first derivative of ${ }^{M_{\delta}(g)}$ :

$\left\langle\frac{d M_{\delta}(g)}{d g}, \tilde{g}\right\rangle=\lim _{t \rightarrow 0} \frac{M_{\delta}(g+t \tilde{g})-M_{\delta}(g)}{t} \forall \tilde{g} \in L^{2}\left(I_{0}\right)^{d}$

where

$$
\begin{aligned}
M_{\delta}(\bar{g})-M_{\delta}(g) & =J_{\delta}\left(\overline{u_{1}}, \overline{u_{2}}, \bar{g}\right)-J_{\delta}\left(u_{1}, u_{2}, g\right) \\
& =\int_{\Gamma_{0}}\left(\frac{1}{2}\left(\left(\overline{u_{1}}-\overline{u_{2}}\right)^{2}-\left(u_{1}-u_{2}\right)^{2}\right)+\frac{\delta}{2}\left(\bar{g}^{2}-g^{2}\right)\right) d \Gamma_{0}
\end{aligned}
$$

$\bar{g}=g+t \tilde{g}, \bar{u}_{i}$ are solutions of

$a_{\rho_{i}}\left(\bar{u}_{u}, v_{i}\right)=\left(f, v_{i}\right)_{\Omega_{i}}+(-1)^{i+1}\left(g+t \tilde{g}, v_{i}\right)_{\Gamma_{0}}$

$=\left(f, v_{i}\right)_{\Omega_{i}}+(-1)^{i+1}\left(g, v_{i}\right)_{\Gamma_{0}}+(-1)^{i+1} t\left(\widetilde{g}, v_{i}\right)_{\Gamma_{0}}=a_{\rho_{i}}\left(u_{i}, v_{i}\right)+(-1)^{i+1} t\left(\widetilde{g}, v_{i}\right)_{\Gamma_{0}}$

which implies that

$a_{\rho_{i}}\left(\overline{u_{i}}-u_{i}, v_{i}\right)=(-1)^{i+1} t\left(\tilde{g}, v_{i}\right)_{\Gamma_{0}}$

then 
$a_{\rho_{i}}\left(\frac{\overline{u_{i}}-u_{i}}{t}, v_{i}\right)=(-1)^{i+1}\left(\widetilde{g}, v_{i}\right)_{\Gamma_{0}} \quad \forall v_{i} \in H_{\Gamma_{i}^{i}}^{1}\left(\Omega_{i}\right)^{d}$

put

$\tilde{u}_{i}=\frac{\overline{u_{i}}-u_{i}}{t}$

$a_{\rho_{i}}\left(\tilde{u}_{i}, v_{i}\right)=(-1)^{i+1}\left(\tilde{g}_{i} v_{i}\right)_{\Gamma_{0}} \forall v_{i} \in H_{\Gamma_{i}}^{1}\left(\Omega_{i}\right)^{d}$

by replacing (18) in (17) we have:

$\frac{M_{\delta}(g+t \tilde{g})-M_{\delta}(g)}{t}=\int_{\Gamma_{0}}\left(\left(\tilde{u}_{1}-\tilde{u}_{2}\right)\left(u_{1}-u_{2}\right)+t\left(\tilde{u}_{1}-\tilde{u}_{2}\right)^{2}+\frac{\delta t}{2} \tilde{g}^{2}+\delta g \tilde{g}\right) d \Gamma_{0}$

thus

$\left(\frac{d M_{\delta}(g)}{d g}, \tilde{g}\right)=\int_{\Gamma_{0}}\left(\left(\tilde{u}_{1}-\tilde{u}_{2}\right)\left(u_{1}-u_{2}\right)+\delta g \tilde{g}\right) d \Gamma_{0} \quad \forall \tilde{g} \in L^{2}\left(\Gamma_{0}\right)^{d}$

Set $^{v_{i}=\lambda_{i}}$ in (19), $\zeta=\tilde{u}_{i}$ in (12)

$\Rightarrow$

$\left(\tilde{g}, \lambda_{1}-\lambda_{2}\right)_{\Gamma_{0}}=\left(\tilde{u}_{1}-\tilde{u}_{2}, u_{1}-u_{2}\right)_{\Gamma_{0}}$

consequently (20) becomes:

$\left\langle\frac{d M_{\delta}(g)}{d g}, \tilde{g}\right\rangle=\int_{\Gamma_{0}}\left(\tilde{g}\left(\lambda_{1}-\lambda_{2}\right)+\delta g \tilde{g}\right) d \Gamma_{0} \quad \forall \tilde{g} \in L^{2}\left(\Gamma_{0}\right)^{d}$

which yields an explicit formula for the gradient

$\frac{d M_{\delta}(g)}{d g}=\delta g+\left(\lambda_{1}-\lambda_{2}\right) / \Gamma_{0}$

The simple gradient method is defined as follows:

$g^{(n+1)}=g^{(n)}-\frac{\alpha}{\delta} \frac{d M_{\delta}(g)}{d g} \quad$ for $n=1,2, \ldots$

where $\frac{\bar{\alpha}}{\bar{\delta}}$ is a step size. Combining with (21) yields a formula to update $g$ :

$g^{(n+1)}=(1-\alpha) g^{(n)}-\frac{\alpha}{\delta}\left(\lambda_{1}^{(n)}-\lambda_{2}^{(n)}\right)$

Hence following the discussion above the proposed algorithm to solve (5) is:

\section{Algorithm}

The heading Make an initial guess for the density $\rho$ on the overall domain $\Omega$, then the corresponding $\rho_{i}=\rho / \Omega_{i}$ in each subdomain $\Omega_{i}(i=1,2)$ satisfies: $\rho_{1}=\rho_{2}$ on $\Gamma_{0}$.

repeat

For each ${ }^{\rho_{i}}$, compute the resulting displacements, a loop on ${ }^{g}$ ensures that $\rho_{1}=\rho_{2}$ on $\Gamma_{0}$

Given a starting guess $g^{(0)}$

for $n=0,1_{s}, \ldots$ do 
repeat

determine $u_{i}^{(n)},(i=1,2)$ from

$a_{\rho_{i}}\left(u_{i}^{(n)}, v_{i}\right)=\left(f, v_{i}\right)_{\Omega_{i}}+(-1)^{i+1}\left(g^{(n)}, v_{i}\right)_{\Gamma_{0}} \quad \forall v_{i} \in H_{\Gamma_{i}^{i}}^{1}\left(\Omega_{i}\right)^{d}$

determine $\lambda_{i}^{(n)},(i=1,2)$ from

$a_{\rho_{i}}\left(\xi, \lambda_{i}\right)=(-1)^{i+1}\left(u_{1}^{(n)}-u_{2}^{(n)}, \xi\right)_{\Gamma_{0}} \quad \forall \xi \in H_{\Gamma_{0}}^{1}\left(\Omega_{i}\right)^{d}$

determine $g^{(n+1)}$ from

$g^{(n+1)}=(1-\alpha) g^{(n)}-\frac{\alpha}{\delta}\left(\lambda_{1}^{(n)}-\lambda_{2}^{(n)}\right)$

until a stopping criteria is satisfied

end for

Compute the compliance and sensitivities in each subdomain

Mesh independency filter in $\Omega$ [8]

Update design variables in $\Omega(15)$

until the change in design variables is less than $1 \%$ (this stopping criteria could be decreased

if needed) [8, p.263].

\subsection{Numerical results}

The numerical test of the proposed algorithm is the beam (see figure 1).

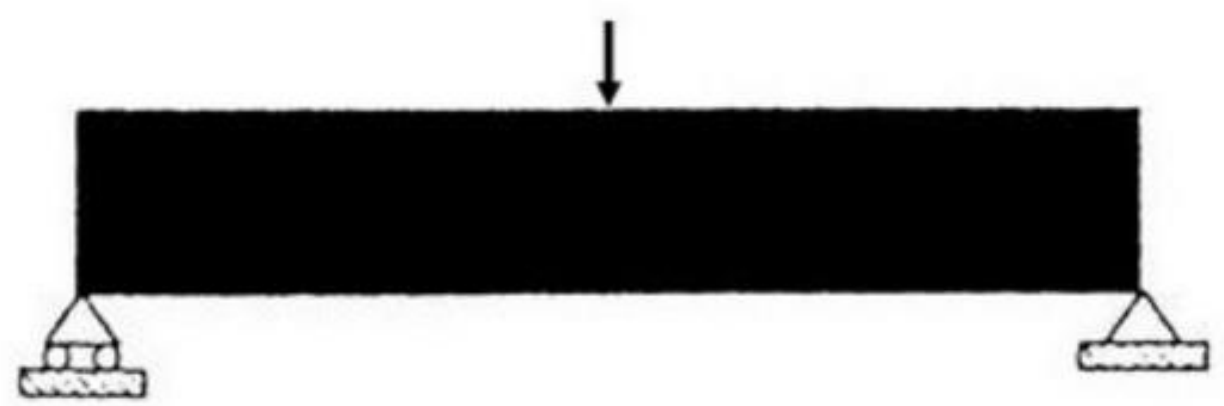

Fig 1: Design domain with boundary conditions

The beam is decomposed in two equal beams. Figure 2 gives the result of the direct topology optimization of this structure using the Matlab code proposed in [8].

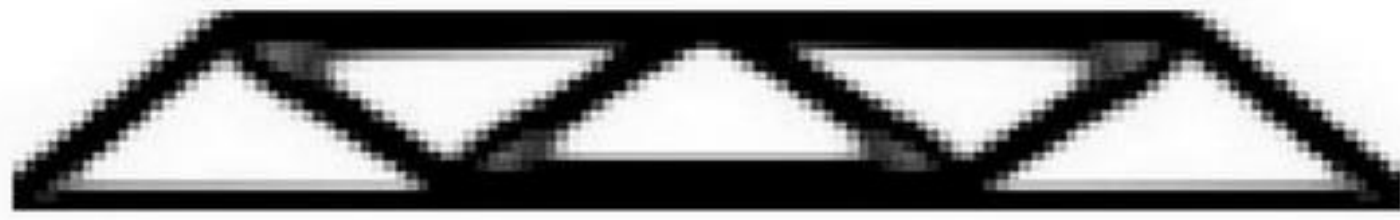

Fig 2: Topology optimization of the structure

Figures 3 and 4 give the result of the proposed method. The stop test is based on the maximum norm and equal to $10^{-6}$. 


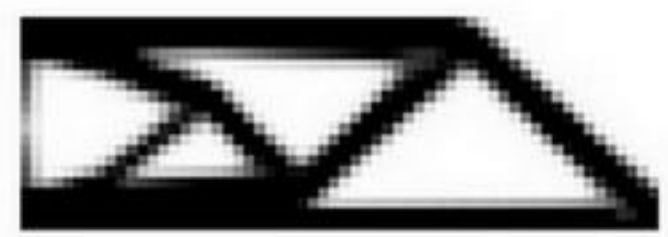

Fig 3: The right part of the structure

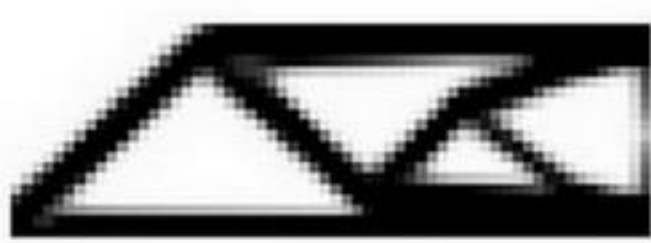

Fig 4: The left part of the structure

The algorithm converges in three iterations. One can show that if the two substructures are assembled, we don't find the result of the figure 3 form the initial structure, because the uniqueness of the solution of the topology optimization is not shown in any reference. So, the finding result stay reasonable and good.

\section{CONCLUSION}

Using a bilevel analysis of the decomposed topology optimization problem, we have developed a new method to solve this complicated problem and the numerical results show her efficiency. In the future work, we want to do numerical implementation of this new algorithm for complex structures issue from the industry to show her efficiency.

\section{REFERENCES}

[1] A. Makrizi, B. Radi and A. El Hami, Solution of the Topology Optimization Problem Based Subdomains Method, Applied Mathematical Sciences, Vol. 2, No. 41, 2008, pp. 2029-2045.

[2] A. Makrizi and B. Radi, Multilevel Approach of a Decomposed Topology Optimization Problem, $9^{\text {èmes }}$ Journées d'Analyse Numérique et d'Optimisation, FST, Mohammedia(Morocco), 17-19 December 2008.

[3] P. Duysinx, Optimisation topologique: du milieu continu à la structure élastique, Ph.D. Thesis, Université de Liège, Faculté des Sciences Appliquées, 7 February 2007.

[4] K. Shimizu, Y. Ishizuka and J. F. Bard, Nondifferentiable and Two-Level Mathematical Programming, Kluwer Academic Publishers, Boston, 1997.

[5] Z. Q. Luo, J. S. Pang and D. Rulph, Mathematical Programs with Equilibrium Constraints, Cambridge University Press, 1996.

[6] J. V. Outrata, M. Kocvara and J. Zowe, Nonsmooth Approach to Optimization Problems with Equilibrium Constraints, Kluwer Academic Publishers, 1998.

[7] A. Evgrafov, Approximation of Topology Optimization Problems using Sizing Optimization Problems, Ph.D. Thesis, Chalmers University of Technology and Göteborg University, Göteborg, Sweden, 2004.

[8] M. P. Bendsoe and O. Sigmund, Topology Optimization, Theory, Methods, and Applications, Springer Verlag, 2003. 


\section{Author' biography with Photo}

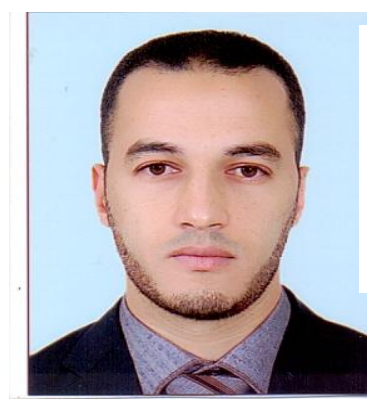

Dr. A. Makrizi, actually Assistant Professor of Mathematics and Mathematics Education at the Regional Center for the professions of education and training. He has published 5 research papers in topology optimization.

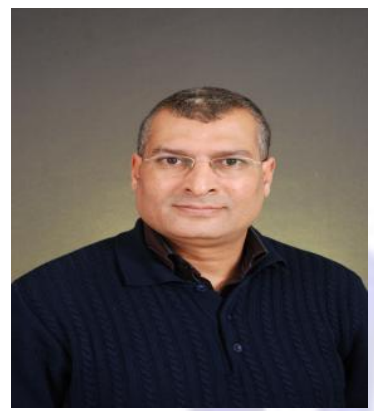

Phd Thesis in Applied Mathematics and actually Professor at the University Hassan Premier. Pr. B. Radi is author of three scholar books and many papers in scientific reviews.

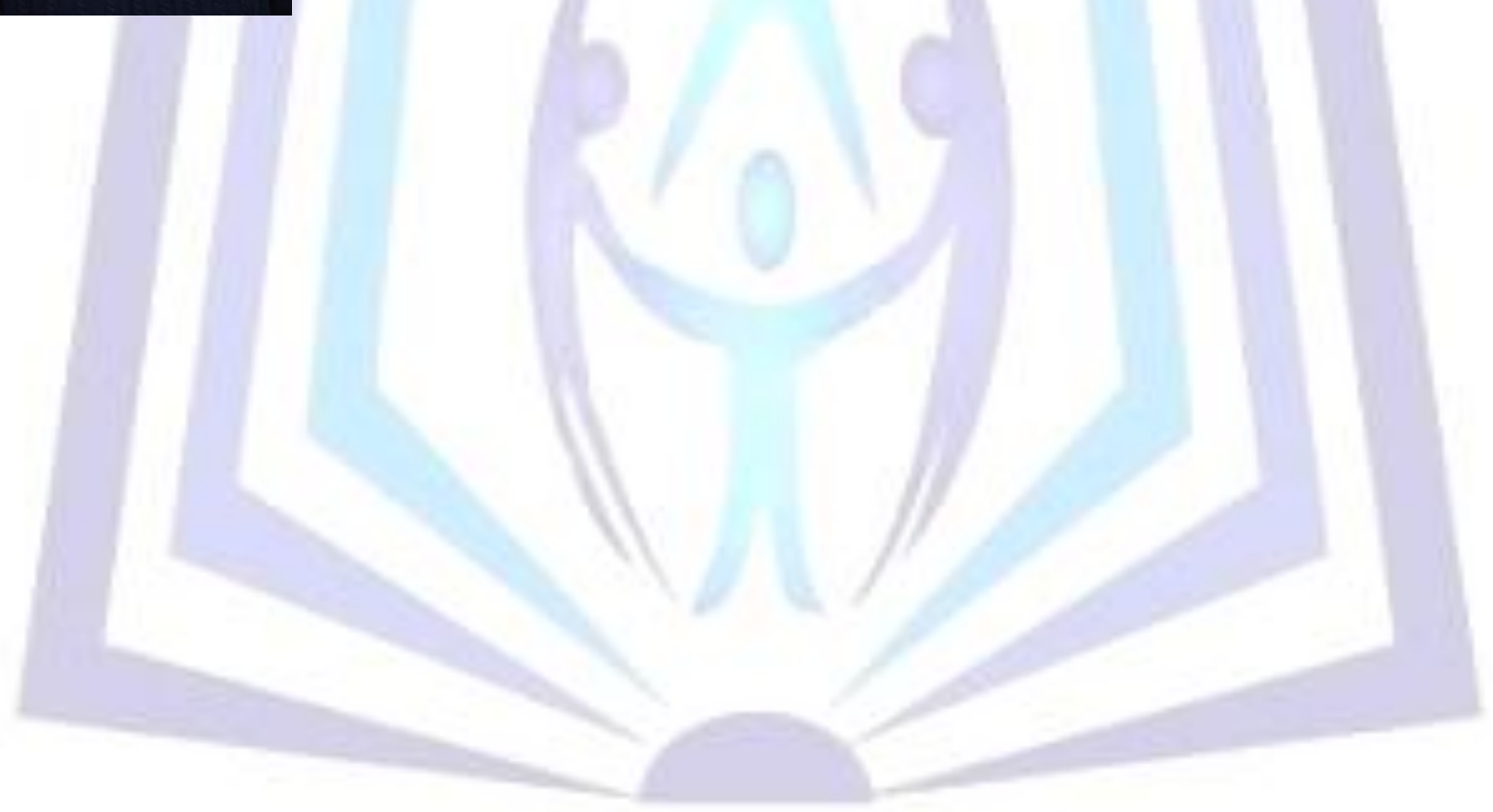

